\title{
Call for Papers: Special Issue of Earth, Planets and Space (EPS) "High Energy Earth Science: Muon and Neutrino Radiography"
}

For the purpose of radiography, recent developments in muon and neutrino detectors make it possible to detect the muon and neutrino particles with high statistics and therefore in great detail. Because of their relevance for a variety of related fields of research, neutrino and muon physics will be a major focus in geophysical research. It is likely that several crucial questions concerning geodynamics and related fields such as volcanology and tectonics will be answered. The successful operation of such detectors, therefore, may very well represent the birth of a new technique for the study of fundamental issues in these fields.

The 1st International workshop on high energy earth science; muon and neutrino radiography is held on 26-27 June 2008: http://www.eri.u-tokyo.ac.jp/ht/workshop08/index.html. The workshop is devoted to addressing the main current issues in this research area to form a research network aimed at fostering crossdisciplinary scientific collaboration on neutrino physics, muon phyics, positron physics and geophysics. The purpose of the workshop is to develop a new geophysical insight into recent progress in muon and neutrino radiography design, the associated R\&D program, and evolving geological and geophysical program that motivates high energy earth science.

In view of the great success of the workshop, we propose a proceeding of the International Workshop on High Energy Earth Science as a special issue of Earth, Planets and Space. The publication will not be limited to papers presented in the meeting and, therefore, we encourage contributions from anybody interested. Contributors to this special issue should submit their papers to the EPS editorial office either by post or by e-mail (eps@terrapub.co.jp), following the instructions for regular EPS submission. For details, please visit the following web page:

http://www.terrapub.co.jp/journals/EPS and click on "Information for Contributors".

Please state "For Special issue for the High energy earth science: muon and neutrino radiography" clearly in the covering letter that accompanies the submission. We encourage electronic submissions. After papers are accepted, the authors will receive instructions for the final manuscript from the editorial office. The deadline for manuscript submissions for this special issue is 31 October 2008. This special issue will hopefully be published in early 2009.

For more information on this special issue, please contact the corresponding editor (Hiroyuki Tanaka, ht@riken.jp); questions on manuscript preparation should be addressed to the EPS editorial office.

Note: EPS accepts manuscripts of original research contributions only, and so-called "review papers" will not be accepted. EPS will review all the manuscripts before the publication.

Guest Editors: Hiroyuki Tanaka, University of Tokyo, Japan

Francis Halzen, University of Wisconsin, Madison, USA or

Thomas K. Gaisser, University of Delaware, USA

Albert Tarantola, Institut de Physique du Globe de Paris, France

Paolo Strolin, Universita di Napoli Federico II, Italy 